\title{
The female advantage: sex as a possible protective factor against emotion recognition impairment following traumatic brain injury
}

\author{
Arianna Rigon $^{1} \cdot$ Lyn Turkstra $^{2} \cdot$ Bilge Mutlu $^{3} \cdot$ Melissa Duff $^{1,4,5}$
}

Published online: 31 May 2016

(C) Psychonomic Society, Inc. 2016

\begin{abstract}
Although moderate to severe traumatic brain injury (TBI) leads to facial affect recognition impairments in up to $39 \%$ of individuals, protective and risk factors for these deficits are unknown. The aim of the current study was to examine the effect of sex on emotion recognition abilities following TBI. We administered two separate emotion recognition tests (one static and one dynamic) to 53 individuals with moderate to severe TBI (females $=28$ ) and 49 demographically matched comparisons (females $=22$ ). We then investigated the presence of a sex-by-group interaction in emotion recognition accuracy. In the comparison group, there were no sex differences. In the TBI group, however, females significantly outperformed males in the dynamic (but not the static) task. Moreover, males (but not females) with TBI performed
\end{abstract}

Electronic supplementary material The online version of this article (doi:10.3758/s13415-016-0437-0) contains supplementary material, which is available to authorized users.

Arianna Rigon

arianna-rigon@uiowa.edu

1 Interdisciplinary Neuroscience Program, The University of Iowa, 420-I Seashore Hall, 328 Iowa Ave., Iowa City, IA 52242, USA

2 Department of Communication Sciences and Disorders, The University of Wisconsin-Madison, 474 Goodnight Hall 1975 Willow Dr., Madison, WI 53706, USA

3 Department of Computer Sciences, The University of WisconsinMadison, 1210 W. Dayton St., Room 6381, Madison, WI 53706-1685, USA

4 Department of Communication Sciences and Disorders, The University of Iowa, 121D Wendell Johnson Speech and Hearing Clinic, 250 Hawkins Dr., Iowa City, IA 52242, USA

5 Department of Neurology, The University of Iowa, 2007 Roy Carver Pavilion, 200 Hawkins Dr., Iowa City, IA 52242, USA significantly worse than comparison participants in the dynamic task. Further analysis revealed that sex differences in emotion recognition abilities within the TBI group could not be explained by lesion location, TBI severity, or other neuropsychological variables. These findings suggest that sex may serve as a protective factor for social impairment following TBI and inform clinicians working with TBI as well as research on the neurophysiological correlates of sex differences in social functioning.

Keywords Traumatic brain injury $\cdot$ Emotion recognition · Facial affect recognition $\cdot$ Sex difference

Social impairment is common in individuals with moderate to severe traumatic brain injury (TBI; Andrews, Rose, \& Johnson, 1998; Duff, Mutlu, Byom, \& Turkstra, 2012; Gomez-Hernandez, Max, Kosier, Paradiso, \& Robinson, 1997; Milders, Fuchs, \& Crawford, 2003; Temkin, Corrigan, Dikmen, \& Machamer, 2009; Ylvisaker, Turkstra, \& Coelho, 2005) and is a major predictor of overall outcome (Morton \& Wehman, 1995). Facial affect recognition ability, in particular, is strongly associated with negative social outcomes, such as poor social integration (Knox \& Douglas, 2009) and reports of socially inappropriate behaviors (Pettersen, 1991).

A recent meta-analysis estimated that up to $39 \%$ of individuals in the chronic phase of a moderate to severe TBI are significantly impaired at recognizing facial affect (Babbage et al., 2011). To date, however, there are no methods for identifying which patients are at risk for emotion recognition deficits. Rosenberg, Dethier, Kessels, Westbrook, and McDonald, (2015) provided some evidence that TBI severity (measured by posttraumatic amnesia) is a risk factor (Rosenberg et al., 2015), but severity accounted for only $37 \%$ of the variance in affect-recognition task scores. 
Another candidate predictor of impairments is site of brain damage, because TBI often affects brain regions implicated in affect recognition. However, Green, Turner, and Thompson (2004) reported that participants with TBI who had focal damage in regions involved in emotion recognition-including right posterior hemisphere, amygdala, basal ganglia-did not perform significantly worse than participants with TBI who had damage in other brain regions (Green et al., 2004). It has been hypothesized that the degree of emotion recognition impairment might correlate with the degree of frontal or fronto-temporal functioning (Bornhofen \& McDonald, 2008; Hornak, Rolls, \& Wade, 1996; Olson, McCoy, Klobusicky, \& Ross, 2013; Willis, Murphy, Ridley, \& Vercammen, 2015). While this claim has some support from the TBI literature (Martins et al., 2012; Spikman, Timmerman, Milders, Veenstra, \& van der Naalt, 2012), findings to date remain inconclusive.

There is some evidence that facial affect recognition abilities are related to other nonsocial cognitive skills, such as verbal and nonverbal working memory and processing speed (Yim, Babbage, Zupan, Neumann, \& Willer, 2013), verbal concept formation and alternating fluency (Williams \& Wood, 2010), and cognitive flexibility (Milders et al., 2003). Other studies, however, have found no correlations between emotion recognition and these skills (Rosenberg et al., 2015; Spikman et al., 2012).

A final potential predictor of emotion recognition impairments is sex of the participant. There is a long history of research showing a female advantage in recognizing emotions (Kret \& De Gelder, 2012; Montagne, Kessels, Frigerio, de Haan, \& Perrett, 2005; Weisenbach et al., 2014) beginning in infancy and continuing throughout the life span (Kessels, Montagne, Hendriks, Perrett, \& de Haan, 2014; McClure, 2000). Thus, women with TBI might have an advantage that would protect them from affect recognition impairments, whereas men with TBI might be more vulnerable. To date, examinations of the relationship between sex and TBI outcome, however, have yielded mixed results. For instance, data from the IMPACT study showed that sex is not a significant predictor of 6-month overall outcome as measured by the Glasgow Outcome Scale (Mushkudiani et al., 2007), but a previous metaanalysis by Farace and Alves (2000) revealed that women with TBI had worse outcome than men. These mixed results are likely to be due to the multiplicity of measures that have been used to assess outcome. Indeed, breaking outcome down into the cognitive domain highlights the complexity of the relationship between cognitive abilities and sex following TBI; women outperform men in language, attention- and working-memory tasks (Ratcliff et al., 2007), and visual memory (Moore, Ashman, Cantor, Krinick, \& Spielman, 2010), whereas the opposite is true for visual analytic skills (Ratcliff et al., 2007).

Recent work on TBI populations has reported sex-based differences in the social domain. Despins, Turkstra, Struchen, and Clark (2015) found that women with TBI rated their pragmatic communication skills more accurately than did men with comparable injury severity. Adult females with TBI are better than males at theory-of-mind tasks (Turkstra, 2008), and they show higher rates of community integration years after their injury (R. L. Wood \& Rutterford, 2006). A longitudinal study on pediatric TBI found that girls not only performed better on a facial affect-recognition task at baseline but also improved their skills at a higher rate than boys (Schmidt, Hanten, Li, Orsten, \& Levin, 2010). These findings, coupled with clinical evidence that women experience better social outcomes than men after TBI (Farace \& Alves, 2000), support the notion that women may have better resilience to TBI-related social deficits. However, there is little experimental evidence for an interaction between sex and brain injury in emotion recognition abilities. Further clarification of the role of sex in social functioning in general, and in facial affect recognition in particular, is crucial; not only does such understanding have the potential to inform clinicians of subgroups of patients with TBI who are more likely to develop emotion recognition impairment and may benefit from early rehabilitation, but it can also inform the development of new behavioral and pharmacological treatments tailored to men or women.

In the current study, we sought to examine the relationship between sex and the ability to label facial affect in adults with TBI. Based on evidence of sex-differences in social functioning in both TBI and healthy populations, we hypothesized that being female would serve as a protective factor for emotion recognition impairment following TBI. To test this hypothesis, we administered two different emotion-labeling tasks to a sample of individuals with moderate to severe TBI and a demographically matched comparison group. The first task was a traditional emotion recognition test, in which participants were asked to label emotions displayed by actors in photographs. The second was a dynamic facial affect-recognition task, in which stimuli included morphed images that presented a range of intensities of emotion expression. Dynamic tasks including different intensities have been argued to be more ecologically valid and to reveal individual differences within healthy populations (Montagne, Kessels, De Haan, \& Perrett, 2007; Wehrle, Kaiser, Schmidt, \& Scherer, 2000). Our main predictions were a main effect of group, resulting in higher accuracy on both tasks in the comparison group, and an interaction of group by sex, manifested as higher overall scores in women and a larger sex-based difference between women and men with TBI. Moreover, we investigated the presence of a three-way interaction among group, sex, and emotion type in order to explore whether the sex-based difference was specific 
to individual emotions. Last, we aimed to determine how sex influences the relationship between emotion recognition abilities and performance on tasks measuring "nonsocial" cognitive skills, such as executive functioning, verbal learning and memory, processing speed, and self-reported psychological distress.

\section{Method}

\section{Participants}

A total of 53 individuals with TBI (female $=25$ ) and 49 healthy comparison participants $(\mathrm{NC})($ female $=27)$ took part in the study. Participants were recruited through ads and brain injury units in Iowa City, Iowa (IA), and Madison, Wisconsin (WI). Inclusionary criteria for individuals with TBI were (1) history of moderate to severe TBI, (2) chronic postinjury phase (i.e., >6-months postinjury), (3) aphasia quotient higher than 93.8 on the Western Aphasia Battery (WAB; Shewan \& Kertesz, 1980), and (4) age between 18 and 65 years. TBI severity was assessed using the Mayo Classification System (Malec et al., 2007; see Supplementary Information for severity details and psychiatric history of participants with TBI). Inclusionary criteria for NC were (1) no history of head injury or loss of consciousness; (2) no history of neurological, psychiatric, or learning disorders; (3) aphasia quotient higher than 93.8 on the WAB; (4) age between 18 and 65 years.

TBI and NC groups were matched for age $(t(100)=-.56, p$ $=.57)$, education $(t(100)=.71, p=.48)$ and $\operatorname{sex}\left(\chi^{2}(1)=.64, p\right.$ $=.42)$. Mean injury chronicity was 14.94 months $(S D=$ 16.33). There were no significant group differences between females with and without TBI for age $(t(50)=-1, p=.32)$ or education $(t(50)=.15, p=.88)$. There were also no significant group differences between males with and without TBI for age $(t(48)=.2, p=.84)$ or education $(t(48)=.64, p=.53)$. Lastly, within both the TBI and the NC group, males and females were matched for age (TBI: $t(51)=-.03, p=.98$; NC: $t(47)$ $=1.1, p=.29)$ and education (TBI: $t(51)=-1.79, p=.08$; NC: $t(47)=-1.57, p=.12)($ see Table 1$)$.

\section{Emotion-recognition tasks}

Two separate facial affect-recognition tasks were administered as part of a larger battery: the Karolinska Directed Emotional Faces test (KDEF) and the Emotion Recognition Test (ERT). The battery was administered in two 2-hour sessions. Administration of both facial affect-recognition tasks occurred in the first session, and the order of the tasks was counterbalanced. Furthermore, the two emotion-recognition tasks never directly followed each other.

The KDEF test (Calvo \& Lundqvist, 2008) is a computerbased emotion-recognition task. The task consists of 28 photos of static facial expressions, including four photographs in which actors (two males and two females) depict each of the six basic emotions (afraid, angry, disgusted, happy, sad, surprised), and four photos of neutral facial expressions (see supplementary information for stimuli information). Participants were presented with a picture and seven response choices listed to the right of the photo (afraid, angry, disgusted, happy, neutral, sad, surprised), and they were asked to indicate the word best describing the person's feeling. The dependent variable was the number of accurate responses for each emotion and the number of accurate responses across all emotions.

The ERT (Kessels et al., 2014) is another computer-based facial affect-recognition task. Instead of static photographs, this task involves videos of faces first appearing neutral then gradually morphing to express one of six basic emotions (afraid, angry, disgusted, happy, neutral, sad, surprised). The long form of the test includes morphs from neutral to nine different levels of emotion intensity (20\% to $100 \%$ at $10 \%$ increments). The short version of the test, which was used in this study, has 96 items and includes morphs from neutral to four levels of emotion intensities ( $0 \%$ to $40 \%, 60 \%, 80 \%$, and $100 \%$ ). The presentation order of morphs is fixed, with incremental increases from lowest to highest emotion intensities. For each trial, participants are presented with the morph and six response choices listed to the right of the stimulus (afraid, angry, disgusted, happy, sad, surprised). For each intensity, 24 trials were administered (four for each emotion, including two with a male actor and two with a female actor) for a total of 96 trials. Participants were instructed to indicate "the word that best describes what the person is feeling." Dependent variables obtained from this task were the number of correct responses for each emotion and intensity, the number of correct responses for each intensity across all emotions, and the total number of correct responses across all intensities and emotions.

\section{Neuropsychological tasks}

In addition to the two emotion-recognition tasks, participants were administered (1) the California Verbal Learning Test (CVLT; Delis, Freeland, Kramer, \& Kaplan, 1988), to assess immediate recall (CVLT-IR), short-delay verbal recall (CVLTSR), and long-delay verbal recall (CVLT-LR); (2) the Trail Making Test (Trails; Gordon, 1972), to assess executive functioning; (3) the Coding and Symbol Search subtests of the Wechsler Adult Intelligence Scale (Holdnack, Xiaobin, Larrabee, Millis, \& Salthouse, 2011), in order to obtain a processing speed index (PSI); and (4) the Brief Symptom Inventory (BSI-18) (Derogatis \& Melisaratos, 1983), to evaluate psychological distress and psychiatric disorders on dimensions of Somatization, Depression, Anxiety, and Global Severity Index. Due to the longitudinal nature of the study, 
Table 1 Comparison of demographic variables between and within NC and TBI groups

\begin{tabular}{|c|c|c|c|c|c|c|c|c|c|c|c|}
\hline \multirow[t]{2}{*}{ Variable } & \multicolumn{3}{|l|}{$\begin{array}{l}\mathrm{NC} \\
M(S D)\end{array}$} & \multicolumn{3}{|l|}{$\begin{array}{l}\text { TBI } \\
M(S D)\end{array}$} & \multicolumn{5}{|c|}{$\begin{array}{l}\text { Group Differences } \\
(p)\end{array}$} \\
\hline & Total & Females & Males & Total & Females & Males & $\begin{array}{l}\text { TBI vs. } \\
\text { NC }\end{array}$ & $\begin{array}{l}\text { TBI } \\
\text { (Males vs. } \\
\text { Females) }\end{array}$ & $\begin{array}{l}\text { NC } \\
\text { (Males vs. } \\
\text { Females) }\end{array}$ & $\begin{array}{l}\text { Females (TBI } \\
\text { vs. NC) }\end{array}$ & $\begin{array}{l}\text { Males } \\
\text { (TBI vs. } \\
\text { NC) }\end{array}$ \\
\hline $\begin{array}{l}N / \text { Sex } \\
\text { composi- } \\
\text { tion }\end{array}$ & 49 & 27 & 22 & 53 & 25 & 28 & .42 & N/A & N/A & N/A & N/A \\
\hline Age & $\begin{array}{l}42.54 \\
\quad(14.47)\end{array}$ & $\begin{array}{l}40.55 \\
(13.58)\end{array}$ & $\begin{array}{l}44.98 \\
\quad(15.46)\end{array}$ & $\begin{array}{l}44.14 \\
\quad(14.08)\end{array}$ & $\begin{array}{l}44.21 \\
\quad(12.59)\end{array}$ & $\begin{array}{l}44.09 \\
\quad(15.52)\end{array}$ & .57 & .98 & .29 & .32 & .84 \\
\hline Education & $\begin{array}{l}15.09 \\
(1.77)\end{array}$ & $\begin{array}{l}15.44 \\
(1.86)\end{array}$ & $\begin{array}{c}14.66 \\
(1.58)\end{array}$ & $\begin{array}{l}14.81 \\
(2.15)\end{array}$ & $\begin{array}{l}15.36 \\
(2.2)\end{array}$ & $\begin{array}{c}14.32 \\
(2.02)\end{array}$ & .48 & .08 & .12 & .88 & .53 \\
\hline Site & $\begin{array}{l}\mathrm{WI}=26 ; \\
\mathrm{IA}=23\end{array}$ & $\begin{array}{l}\mathrm{WI}=9, \\
\mathrm{I}- \\
\mathrm{A}=13\end{array}$ & $\begin{array}{l}\mathrm{WI}=17 \\
\mathrm{IA}=10\end{array}$ & $\begin{array}{l}\mathrm{WI}=30 \\
\quad \mathrm{IA}=23\end{array}$ & $\begin{array}{l}\mathrm{WI}=16, \\
\mathrm{IA}=12\end{array}$ & $\begin{array}{l}\mathrm{WI}=14, \\
\quad \mathrm{IA}=11\end{array}$ & .71 & .93 & .15 & .39 & .78 \\
\hline
\end{tabular}

Note $\mathrm{NC}=$ normal comparison participants, $\mathrm{TBI}=$ traumatic brain injury, $\mathrm{IA}=\mathrm{Iowa}, \mathrm{WI}=\mathrm{Wisconsin}, \mathrm{N} / \mathrm{A}=$ not available

some measures were missing for a subset of the participants: CVLT (missing for four participants with TBI), Trails (missing for four participants with TBI), and PSI (missing for two individuals with TBI). Two participants with TBI were unable to finish Part B of Trails. In these cases, we used a truncation approach, and their Trails B scores were recoded into the lowest $z$ scores found in the overall dataset (Costa, 2014).

\section{Statistical analysis}

Statistical analysis was carried out using IBM SPSS Statistic (Version 21.0). To test the main effect of group, group-by-sex interaction, and interactions among sex, group, and emotion type, we performed a repeated-measures analysis of covariance (ANCOVA) with sex (males vs. females) and group (TBI vs. NC) as between-subjects factors, emotion type (anger, disgust, fear, happiness, neutral, sadness, surprise) as withinsubjects factor, and testing site (Iowa City, IA, vs. Madison, WI) and age as covariates. The latter was included as a covariate because of the extensive literature reporting age differences in facial affect recognition (Montagne et al., 2007).

For ERT data, we investigated the presence of an interaction between sex and group and interactions between sex, group, emotion type, and emotion intensity. We used a repeated-measures ANCOVA with sex (male vs. female) and group (TBI vs. NC) as between-subjects factors and intensity $(40 \%, 60 \%, 80 \%$, and 100\%) and emotion type (anger, disgust, fear, happiness, sadness, surprise) as within-subjects factors. Testing site and age were included as covariates.

Post hoc analyses were carried out using independent samples $t$ tests. Given our hypothesis that having suffered a TBI and being male would lead to lower facial affect recognition abilities, all $t$ tests examining sex or group differences were one-tailed. When multiple comparisons were performed, Bonferroni correction was applied.

For CVLT, Trails, PSI, and BSI, one-tailed independentsamples $t$ tests were carried out to investigate differences between TBI and NCs, and two-tailed independent-samples $t$ tests were performed to explore differences between males and females within the TBI group. Bonferroni correction was applied when necessary. To examine the correlations between emotion recognition abilities and verbal learning and memory, processing speed, executive functioning, and psychological well-being, we computed within-group Pearson correlations using overall KDEF and ERT scores.

\section{Results}

\section{KDEF}

A repeated-measures ANCOVA revealed a significant main effect of group, $F(1,96)=11.56, p=.001, \eta_{\mathrm{p}}{ }^{2}=.107$, and sex, $F(1,96)=4.36, p=.04, \eta_{\mathrm{p}}{ }^{2}=.04$, with NCs performing better overall than TBIs (NC: $M=26.18, S D=1.56$; TBI: $M=24.45$, $S D=3.01)$, and females showing higher facial affectrecognition accuracy than males (females: $M=25.85, S D=$ 1.98; males: $M=24.7, S D=2.96)$. There were also significant effects of age, $F(1,96)=5.65, p=.02, \eta_{\mathrm{p}}{ }^{2}=.06$, with performance declining as age increased (bivariate correlation between age and KDEF score: $r=-.19, p=.03$ ) and emotion, $F(3.3,31679)=4.82, p=.002, \eta_{\mathrm{p}}{ }^{2}=.05$, (Anger: $M=3.49$, $S D=.88$; Disgust: $M=3.65, S D=.72$; Fear: $M=2.63, S D=$ 1.15; Happiness: $M=3.91, S D=.31$; Neutral: $M=3.79, S D=$ .5 ; Sadness: $M=3.8, S D=.51$; Surprise: $M=3.83, S D=.5$ ). The effect of site was nonsignificant, $F(1,96)=1.1, p=.3$, 
$\eta_{\mathrm{p}}{ }^{2}=.01$. There was no sex by group interaction, $F(1,96)=$ $1.46, p=.23, \eta_{\mathrm{p}}{ }^{2}=.02$. The only significant interactions were group by emotion, $F(3.3,31679)=3.24, p=.02, \eta_{\mathrm{p}}{ }^{2}=.03$, and sex by emotion, $F(3.3,31679)=3.52, p=.01, \eta_{\mathrm{p}}{ }^{2}=.4$.

We explored the group-by-emotion interaction by conducting $t$ tests for each emotion (alpha level was set at $.05 / 7=.007)$. Participants with TBI performed worse than NCs on anger, $t(77.17)=3.31, p=.0005$, and disgust, $t(61.88)=3.91, p=.0001$, but not on other emotions (all $t \mathrm{~s}$ $<1.95$, all $p \mathrm{~s}>.02$; see Fig. 1).

Analysis of the sex-by-emotion interaction showed that females were better than males at recognizing fearful expressions, $t(100)=-2.69, p=.004$, but they were not significantly different from males for other emotions (all $t \mathrm{~s}<-2.28$, all $p \mathrm{~s}>$ 01).

\section{ERT}

ERT data analysis revealed significant main effects of group, $F(1,96)=15.98, p=.0001, \eta_{\mathrm{p}}{ }^{2}=.14$, and sex, $F(1,96)=$ $13.08, p=.0004, \eta_{\mathrm{p}}{ }^{2}=.12$, with higher overall ERT scores for NCs $(\mathrm{NC}: M=59.23, S D=8.03$; TBI: $M=52.17, S D=9.4)$ and females (females: $M=58.79, S D=7.96$; males: $M=52.2$, $S D=9.71)$. Site and age effects were nonsignificant, all $F(1$, $96)<2.88, p>.09, \eta_{\mathrm{p}}{ }^{2}<.03$. As we hypothesized, there was a significant sex-by-group interaction, $F(1,96)=4.19, p=.04$, $\eta_{\mathrm{p}}{ }^{2}=.04$. Within-subjects effects analysis also showed significant main effects of emotion, $F(4.45,426.84)=40.87, p<$ $.000001, \eta_{\mathrm{p}}{ }^{2}=.20$ (Anger: $M=10.79, S D=3.15$; Disgust: $M$ $=10.23, S D=3.03$; Fear: $M=4.59, S D=2.93$; Happiness: $M$ $=15.26, S D=1.19$; $S D=.5$; Sadness: $M=6.53, S D=3.11$; Surprise: $M=7.51, S D=2.71)$ and intensity, $F(3,288)=9.42$, $p=.000006, \eta_{\mathrm{p}}{ }^{2}=.09$ (40\%: $M=10.96, S D=2.74 ; 60 \%: M=$ $13.68, S D=2.54 ; 80 \%: M=14.85, S D=3.03 ; 100 \%: M=$ $15.43, S D=3.32)$, including several significant interactions: emotion by group, $F(4.44,426.84)=4.06, p=.001, \eta_{\mathrm{p}}{ }^{2}=.04$, emotion by intensity, $F(11.45,1099.5)=2.93, p=.0006, \eta_{\mathrm{p}}{ }^{2}$ $=.03$, and emotion by intensity by group, $F(11.45,1099.49)=$ $1.8, p=.047, \eta_{\mathrm{p}}{ }^{2}=.02$.

We first examined the emotion-by-intensity-by-group interaction. After correcting for multiple comparisons (alpha level was set at .05/24 =.002), we found that individuals with TBI performed significantly worse than NCs only for specific emotions and intensities: sadness at the $40 \%, 60 \%$, and $100 \%$ intensities, all $t(100)>2.95$, all $p \mathrm{~s}<.002$, anger at the $60 \%$ and $100 \%$ intensities, all $t(100)>2.96$, all $p \mathrm{~s}<.002$, and disgust at the $80 \%$ and $100 \%$ intensities, all $t(100)>3.17$, all $p \mathrm{~s}<.001$.

We then used an independent-samples $t$ test to examine the sex-by-group interaction. As emotion type and intensity did not significantly interact with sex or with sex and group (all $F \mathrm{~S}$ $<1.3, p>.24, \eta_{\mathrm{p}}{ }^{2}<.01$ ), we used overall ERT accuracy (i.e., the sum of all correct responses across all emotions and intensities) as dependent variable. Compared to male NCs, male individuals with TBI had significantly lower ERT scores, $t(48)=4.06, p=.0002$. On the other hand, female individuals with TBI did not significantly differ from female controls, $t(50)=1.62, p=.11$. Similarly, although in the control group male and female ERT performances were not significantly different, $t(47)=-1.2, p=.23$, females in the TBI group had significantly higher scores than males in this group, $t(51)=-$ 3.97, $p=.0002$ (see Fig. 2).

We next examined whether sex differences in emotion recognition could be related to injury-related factors. Within the TBI group, male and female participants did not significantly differ for cause of injury, $\chi^{2}(5)=1.26, p=.94$, chronicity, $t(51)=.32, p=.75$, posttraumatic amnesia, $t(17)=.61, p=$ .55 , loss of consciousness, $t(20)=.89, p=.37$, or Glasgow Coma Scale, $t(24)=.82, p=.42$, but we note that these measures were only available for part of the sample. Because the frontal, temporal, and occipital lobes have been argued to play a role in emotion recognition, we conducted a two-tailed chisquared test to ensure that the amount of participants having frontal, temporal, or occipital lesions did not significantly differ between male and female groups, $\chi^{2}(1)=.73, p=.41$. Last, there was no significant difference in lesion laterality (i.e., left, right, or bilateral) $\chi^{2}(2)=.14, p=.93$.

\section{Neuropsychological tests: group comparison}

Participants with TBI performed significantly worse than NCs in all CVLT subtests, all $t(96)>5.98$, all $p \mathrm{~s}<.000001$, and had significantly lower PSI scores, $t(98)=4.81, p=.000003$. This finding indicates that participants with TBI had lower verbal learning rates as well as verbal memory and processing speed than NCs. However, performance on the Trails, which measures executive functioning, was not significantly different between the two groups, $t(96)=1.65, p=.05$. Regarding the BSI, the test used to asses psychological well-being, individuals with TBI had significantly higher scores than NCs on the somatization scale, the depression scale, and the global scale, all $t(100)>4.2$, all $p$ s $<.00003$. However, the intergroup difference on the anxiety scale was not significant, $t(100)=2.03, p=.02$ (see Supplementary Information, Table S1).

$\mathrm{NC}$ men did not perform significantly differently from $\mathrm{NC}$ women on any of the tests (all $t \mathrm{~s}<1.6$, all $p \mathrm{~s}>.13$; see Supplementary Information, Table S1). Within the TBI group, we found no significant differences between the performances of male and female participants for CVLT, Trails, PSI, or BSI tests (all $t \mathrm{~s}<2.51$, all $p \mathrm{~s}>.02$ ). However, there was a marginal difference in PSI scores, $t(49)=2.03 p=.05$, and in the somatization scale of the BSI, $t(38.56)=2.51, p=.02$, with female participants with TBI showing higher scores for both tests. These marginal differences indicate a trend for female individuals with TBI to report more somatization than their 
Fig. 1 Group differences in recognition accuracy for different emotions on the KDEF test. A repeated-measures ANCOVA revealed a significant interaction between group and emotion type. Post hoc $t$ tests showed that individuals with TBI were worse than NCs at recognizing disgust and anger, but not happiness, surprise, sadness, fear, or neutral expressions. The asterisk $(*)$ denotes a statistically significant difference $(p<.05)$ after Bonferroni correction for multiple comparison
Group differences in recognition accuracy for different emotions on the KDEF test

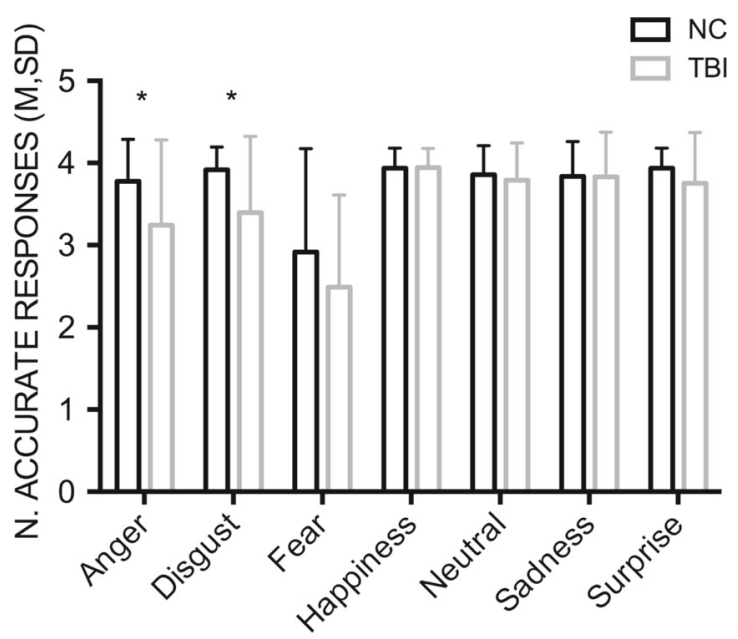

EMOTION TYPE male counterparts and to have higher processing speed than their male counterparts. The findings suggest that even though female participants with TBI showed markedly superior performance in the ERT than male participants, female and male scores on other neuropsychological tests did not differ significantly (see Supplementary Information, Table S1).

\section{Neuropsychological tests and emotion recognition tests: correlations}

To explore the correlation between emotion recognition abilities and psychological distress as well as neuropsychological performance within the TBI group, we calculated Pearson correlations between overall ERT and KDEF scores and scores in CVLT, PSI, Trails, and BSI. For the KDEF, we found that, when correcting for multiple comparisons, overall emotion recognition accuracy was only significantly and positively correlated with PSI ( $r=.36, n=51, p=.005)$. However, there were marginal, positive correlations with BSI anxiety scores $(r=.26, n=53, p=.03)$ and with CVLT-IR $(r=.27$, $n=48, p=.03$ ). Results were similar for ERT scores; although the only significant correlation between overall ERT score was with PSI $(r=.39, n=51, p=.002)$, there were marginal, positive correlations with the somatization $(r=.27, n=53, p$ $=.03)$ and the anxiety $(r=.26, n=53, p=.03)$ scales of the BSI.
Fig. 2 Sex and group differences in emotion-recognition accuracy on the ERT. Post hoc analysis showed that, although there was no significant difference in overall ERT performance between males and females in the NC group, females performed significantly better than males in the TBI group. In addition, females with TBI were not impaired in the ERT compared to NC females; however, male individuals with TBI performed significantly worse than male NCs. The asterisk $\left(^{*}\right)$ denotes statistically significant difference $(p<.01)$

\section{Sex and group differences in emotion recognition accuracy on the ERT}

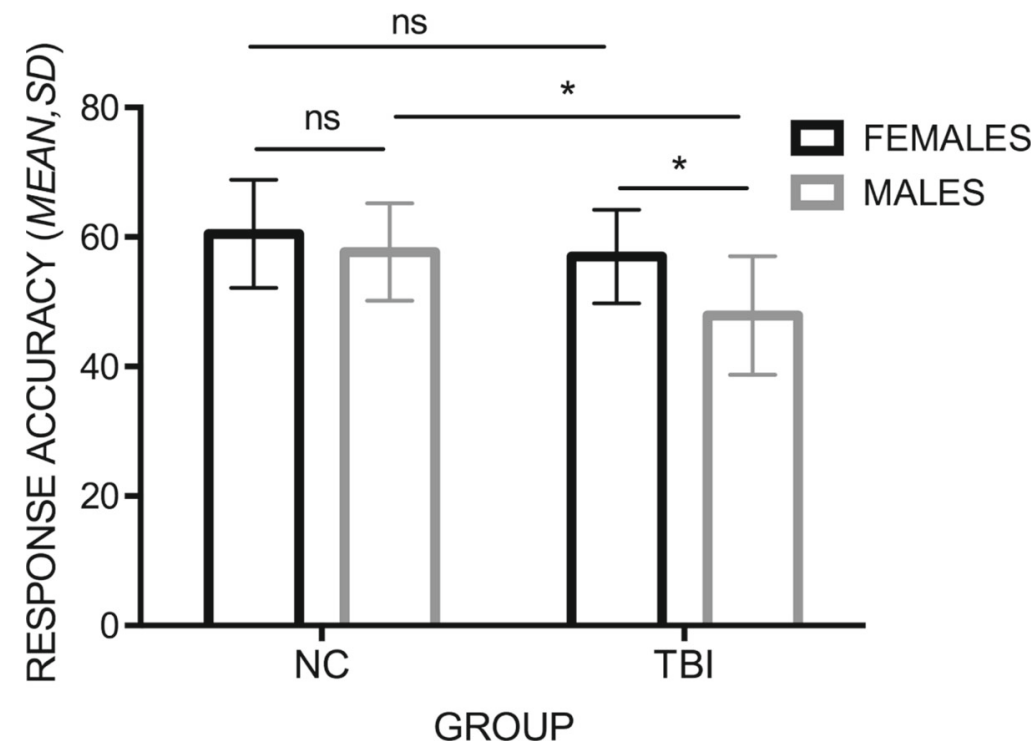


To further explore the role sex differences play in emotion recognition abilities, we calculated separate correlations for male and female participants in the TBI group. Interestingly, we found no significant correlations between KDEF score and any other test within the male TBI group (all $r \mathrm{~s}<.43$, all $n \mathrm{~s}<$ 24 , all $\mathrm{s}>.01$ ) or within the female TBI group (all $r \mathrm{~s}<.32$, all $n \mathrm{~s}<23$, all $p \mathrm{~s}>.06$ ), although male KDEF scores marginally correlated with PSI $(r=.43, n=27, p=.01)$. Similarly, there were no significant correlations between overall ERT score and any other test within the male TBI group (all $r \mathrm{~s}<.44$, all $n \mathrm{~s}<24$, all $p \mathrm{~s}>.01$ ) or within the female TBI group (all $r \mathrm{~s}$ $<.22$, all $n \mathrm{~s}<23$, all $p \mathrm{~s}>.14$ ), although there was a marginal correlation with PSI within the male group $(r=.44, n=27, p=$ .01). Considering that standard deviation values for PSI, ERT, and KDEF were comparable for male and female individuals with TBI (PSI male: $S D=13.24$, female: $S D=17.65$; KDEF male: $S D=3.47$, female: $S D=2.36$; ERT male: $S D=8.74$, female: $S D=6.84$ ) and that neither group's performance showed a ceiling effect, the lack of significant correlations after splitting the TBI group by sex indicates that the association between processing speed and emotion recognition ability was mainly driven by sex differences in processing speed and emotion recognition performance (see Supplementary Information, Table S2).

Last, we examined whether sex differences in processing speed could account for the group-by-sex interaction found for overall ERT performance. A two-way ANCOVA adding PSI as a continuous covariate revealed that, when controlling for processing speed, the group-by-sex interaction remained significant, $F(1,93)=4.6, p=.04, \eta_{\mathrm{p}}^{2}=.05$. This effect indicates that sex differences in emotion recognition abilities could not be explained by group differences in processing speed.

\section{Discussion}

The main goal of this study was to investigate the effects of sex differences on facial affect recognition in adults with TBI. We assessed emotion recognition using two tasks: a static emotion-labeling task and a dynamic task that presents emotions at varying intensities. Based on a large body of evidence indicating female superiority in emotion-recognition tasks within healthy populations (Kret \& De Gelder, 2012; Montagne et al., 2005), we hypothesized that female sex would be a protective factor against facial affect-recognition impairment following TBI. Therefore, we predicted that females who had suffered a TBI would display stronger emotion recognition abilities than their male counterparts.

Although in both emotion-recognition tasks NCs and females performed better than individuals with TBI and males, we found a sex-by-group interaction (i.e., a larger effect of TBI on emotion-recognition performance among male participants) only for the ERT. One possible explanation for this finding is that the full-intensity, static KDEF stimuli were not sensitive enough to detect within-group differences. Indeed, all of the KDEF stimuli were labeled correctly by 12 participants in the NC group $(\sim 24 \%)$ and five in the TBI group $(\sim 9 \%)$. By contrast, no participant in either group correctly responded to $100 \%$ of the ERT trials. The highest score among NC participants was 79 out 96 possible trials ( $82 \%$ accuracy), and the highest score for a participant with TBI was 70 out 96 ( $73 \%$ accuracy). An alternative explanation is that the larger number of items on the ERT increased task difficulty, although this is unlikely because the analysis showed no interaction between intensity and group; that is, group differences were observed even on early items in the task. It is more likely that the lack of a ceiling effect on the ERT allowed within-group variability to emerge, thus revealing the dramatic negative effect of TBI on male emotion-recognition performance.

Men with TBI underperformed both women with TBI and $\mathrm{NC}$ men. This suggests that females with TBI were protected from the potential negative effects of TBI on emotion recognition abilities in virtue of either (a) higher preinjury emotionrecognition abilities that enabled them to perform better postinjury despite having lost the same ground as men, or (b) TBI having a lesser effect on emotion recognition in women than in men. In support of (b), it should be noted that ERT scores in women with TBI were similar to those in female NCs. Moreover, there were no sex differences within the NCs sample; women did not perform significantly better than men, although on average they had higher accuracy. Kessels et al. (2014) used the ERT to examine sex differences in emotion recognition in typical adults and found a female superiority. However, the authors highlighted the high degree of overlap between performances of men and women and the small between-group differences found in their study. It may be that TBI selectively accentuates subtle baseline sex-based differences in cognitive functions, resulting in a better outcome for women in the case of emotion recognition.

Future studies should further explore these sex differences and investigate their underlying mechanisms. For instance, studying the combined effect of sex and TBI on emotion recognition at different points postinjury (e.g., acute/subacute vs. chronic) might help clarify whether these differences are the effect of hormonal or brain-structure-related sex differences (Merz et al., 2012; Veroude, Jolles, Croiset, \& Krabbendam, 2014; Weisenbach et al., 2014; Welborn et al., 2009) versus different responses offered by the social environment to men and women (W. Wood \& Eagly, 2000). Measuring sexhormone levels, even during the chronic phase, might offer information on the relationship between endocrine factors and emotion-recognition abilities. Neuroimaging might provide another helpful tool, both on its own and in combination with hormonal measures. Recent works have attempted to uncover the precise brain regions underlying emotion-recognition 
deficits after TBI by using both task-related functional magnetic resonance imaging approaches (Neumann, McDonald, West, Keiski, \& Wang, 2015) and diffusion tensor imaging approaches (Genova et al., 2015). Although these studies have not examined the effect of sex-based differences, imaging might be able to inform whether emotion-recognition abilities are associated with different white-matter integrity levels or regional activation patterns in uninjured men and women, and to clarify whether brain injury in males preferentially targets these areas. In addition, future studies should further explore the role of gender (i.e., masculinity and femininity) as well as their intersection with sex in emotion recognition impairment secondary to TBI.

Although sex differences were not specific for individual emotions or intensities, we found an interaction between group and emotion on the KDEF and an interaction between group, emotion, and intensity on the ERT. Regarding the KDEF, individuals with TBI performed significantly worse than NCs only on fear, disgust, and anger (three negatively valenced emotions) but not on surprise, sadness, happiness, or neutral faces. Although similar valence-based differences have been widely discussed before (Croker \& McDonald, 2005; Green et al., 2004; Rosenberg, McDonald, Dethier, Kessels, \& Westbrook, 2014), a study by Rosenberg using the ERT showed that individuals with TBI were equally impaired across all types of emotions when task difficulty was controlled across emotions (Rosenberg et al., 2015). Indeed, in our sample, the interaction between group, emotion, and intensity can be explained in a similar way. Although we used the short version of the ERT, so therefore our results are not fully comparable to those obtained by Rosenberg et al. (2015), we found that the differential ability to recognize individual emotions was influenced by the intensity at which they were presented.

Last, when we examined the relationship between emotion recognition abilities and predictors of neuropsychological and psychological well-being within the TBI group, we found that facial affect-recognition skills were positively associated with processing speed. However, when considering males and females with TBI as separate subgroups, we found no correlations between emotion recognition skills and any neuropsychological measure. Although positive correlations between facial affect recognition and processing speed have been reported (Rosenberg et al., 2015; Yim et al., 2013), the association observed in our sample appeared to be driven by sex differences. This finding is notable, especially in the context of the contradictory findings on associations between emotion recognition and "nonsocial" cognitive abilities following TBI. Indeed, it appears that, given the marked sex differences in emotion recognition abilities found in the current study, studies of neuropsychological correlates of facial affect recognition might benefit from treating men and women with TBI as two separate groups or at least including sex as a covariate.
When comparing the current study with previous studies of emotion recognition in adults with TBI, a few factors should be kept in mind. First, our participants had moderate to severe injuries (not only severe injuries), and thus our sample represents individuals with a broader range of injuries. Second, most studies have used different tasks, or different versions of the same task, and this might account for small variations in results across studies (e.g., in the degree of impairment reported for specific emotions). Third, and perhaps most important, as the main purpose of the current study was to examine sex-based differences within TBI populations, more women were included in the sample than in previous studies (Croker \& McDonald, 2005; Green et al., 2004; Rosenberg et al., 2014). Considering that we observed that within TBI populations women tend to perform as well as controls, it is not surprising that data from our TBI group, which was $47 \%$ female, differed from those in previous TBI studies.

In the current study, we could not examine the relationship between severity and the ability to recognize facial expressions of affect. Indeed, one limitation of the study lies in the fact that different types of severity information were available for participants with TBI. The Mayo Classification System (Malec et al., 2007), which allows researchers to indicate TBI severity by combining all available pieces of information, has been extensively used in a wide variety of studies (Baxter et al., 2013; Ham et al., 2014; Rigon, Duff, McAuley, Kramer, \& Voss, 2015). However, as we determined severity by different factors for each participant, within-group comparisons and correlations between TBI severity and behavioral performance were not feasible. Also, the current study focused on the sex of the observer, and influence of sex of the observer should be further examined in future studies.

In conclusion, our study revealed sex-based differences in recognition of facial affect. Our results suggest that sex may explain part of the individual variability widely reported by both researchers and clinicians studying social functioning following TBI. In addition, the results support evidence that facial affect recognition abilities are at least partially independent from other cognitive abilities, such as executive functioning and processing speed, and that they can be selectively impaired in specific subgroups of people with TBI. Overall, our findings highlight the importance of considering specific subpopulations, particularly men with moderate to severe TBI, when developing both assessment and intervention methods for social cognition in individuals with TBI.

Acknowledgments This work was supported by NICHD/NCMRR grant R01 HD071089. 


\section{References}

Andrews, T. K., Rose, F. D., \& Johnson, D. A. (1998). Social and behavioural effects of traumatic brain injury in children. Brain Injury, 12(2), 133-138.

Babbage, D. R., Yim, J., Zupan, B., Neumann, D., Tomita, M. R., \& Willer, B. (2011). Meta-analysis of facial affect recognition difficulties after traumatic brain injury. Neuropsychology, 25(3), 277-285. doi:10.1037/a0021908

Baxter, D., Sharp, D. J., Feeney, C., Papadopoulou, D., Ham, T. E., Jilka, S., . . . Goldstone, A. P. (2013). Pituitary dysfunction after blast traumatic brain injury: The UK BIOSAP study. Annals of Neurology, 74(4), 527-536. doi:10.1002/ana.23958

Bornhofen, C., \& McDonald, S. (2008). Emotion perception deficits following traumatic brain injury: A review of the evidence and rationale for intervention. Journal of the International Neuropsychological Socity, 14(4), 511-525. doi:10.1017/ S1355617708080703

Calvo, M. G., \& Lundqvist, D. (2008). Facial expressions of emotion (KDEF): Identification under different display-duration conditions. Behavior Research Methods, 40(1), 109-115.

Costa, P. J. (2014). Truncated outlier filtering. Journal of Biopharmaceutical Statistics, 24(5), 1115-1129. doi:10.1080/ 10543406.2014.926366

Croker, V., \& McDonald, S. (2005). Recognition of emotion from facial expression following traumatic brain injury. Brain Injury, 19(10), 787-799.

Delis, D. C., Freeland, J., Kramer, J. H., \& Kaplan, E. (1988). Integrating clinical assessment with cognitive neuroscience: Construct validation of the California Verbal Learning Test. Journal of Consulting and Clinical Psychology, 56(1), 123-130.

Derogatis, L. R., \& Melisaratos, N. (1983). The Brief Symptom Inventory: An introductory report. Psychological Medicine, 13(3), 595-605.

Despins, E. H., Turkstra, L. S., Struchen, M. A., \& Clark, A. N. (2015). Sex-based differences in perceived pragmatic communication ability of adults with traumatic brain injury. Archives of Physical Medicine and Rehabilitation. doi:10.1016/j.apmr.2014.06.023

Duff, M. C., Mutlu, B., Byom, L., \& Turkstra, L. S. (2012). Beyond utterances: Distributed cognition as a framework for studying discourse in adults with acquired brain injury. Seminars in Speech and Language, 33(1), 44-54. doi:10.1055/s-0031-1301162

Farace, E., \& Alves, W. M. (2000). Do women fare worse? A metaanalysis of gender differences in outcome after traumatic brain injury. Neurosurgery Focus, 8(1), e6.

Genova, H. M., Rajagopalan, V., Chiaravalloti, N., Binder, A., Deluca, J., \& Lengenfelder, J. (2015). Facial affect recognition linked to damage in specific white matter tracts in traumatic brain injury. Social Neuroscience, 10(1), 27-34. doi:10.1080/17470919.2014.959618

Gomez-Hernandez, R., Max, J. E., Kosier, T., Paradiso, S., \& Robinson, R. G. (1997). Social impairment and depression after traumatic brain injury. Archives of Physical Medicine and Rehabilitation, 78(12), 1321-1326.

Gordon, N. G. (1972). The Trail Making Test in neuropsychological diagnosis. Journal of Clinical Psychology, 28(2), 167-169.

Green, R. E., Turner, G. R., \& Thompson, W. F. (2004). Deficits in facial emotion perception in adults with recent traumatic brain injury. Neuropsychologia, 42(2), 133-141.

Ham, T. E., Bonnelle, V., Hellyer, P., Jilka, S., Robertson, I. H., Leech, R., \& Sharp, D. J. (2014). The neural basis of impaired self-awareness after traumatic brain injury. Brain, 137(Pt. 2), 586-597. doi:10. 1093/brain/awt350

Holdnack, J. A., Xiaobin, Z., Larrabee, G. J., Millis, S. R., \& Salthouse, T. A. (2011). Confirmatory factor analysis of the WAIS-IV/WMS-IV. Assessment, 18(2), 178-191. doi:10.1177/1073191110393106
Hornak, J., Rolls, E. T., \& Wade, D. (1996). Face and voice expression identification in patients with emotional and behavioural changes following ventral frontal lobe damage. Neuropsychologia, 34(4), 247-261.

Kessels, R. P., Montagne, B., Hendriks, A. W., Perrett, D. I., \& de Haan, E. H. (2014). Assessment of perception of morphed facial expressions using the emotion recognition task: Normative data from healthy participants aged 8-75. Journal of Neuropsychology, 8(1), 75-93. doi:10.1111/jnp.12009

Knox, L., \& Douglas, J. (2009). Long-term ability to interpret facial expression after traumatic brain injury and its relation to social integration. Brain Cognition, 69(2), 442-449. doi:10.1016/j.bandc. 2008.09.009

Kret, M. E., \& De Gelder, B. (2012). A review on sex differences in processing emotional signals. Neuropsychologia, 50(7), 12111221. doi:10.1016/j.neuropsychologia.2011.12.022

Malec, J. F., Brown, A. W., Leibson, C. L., Flaada, J. T., Mandrekar, J. N., Diehl, N. N., \& Perkins, P. K. (2007). The Mayo Classification System for Traumatic Brain Injury Severity. Journal of Neurotrauma, 24(9), 1417-1424. doi:10.1089/neu.2006.0245

Martins, A. T., Faisca, L., Esteves, F., Simao, C., Justo, M. G., Muresan, A., \& Reis, A. (2012). Changes in social emotion recognition following traumatic frontal lobe injury. Neural Regeneration Research, 7(2), 101-108. doi:10.3969/j.issn.1673-5374.2012.02.004

McClure, E. B. (2000). A meta-analytic review of sex differences in facial expression processing and their development in infants, children, and adolescents. Psychological Bulletin, 126(3), 424-453.

Merz, C. J., Tabbert, K., Schweckendiek, J., Klucken, T., Vaitl, D., Stark, R., \& Wolf, O. T. (2012). Neuronal correlates of extinction learning are modulated by sex hormones. Social Cognitie and Affective Neuroscience, 7(7), 819-830. doi:10.1093/scan/nsr063

Milders, M., Fuchs, S., \& Crawford, J. R. (2003). Neuropsychological impairments and changes in emotional and social behaviour following severe traumatic brain injury. Journal of Clinical and Experimental Neuropsychology, 25(2), 157-172. doi:10.1076/jcen. 25.2.157.13642

Montagne, B., Kessels, R. P., Frigerio, E., de Haan, E. H., \& Perrett, D. I. (2005). Sex differences in the perception of affective facial expressions: Do men really lack emotional sensitivity? Cognitive Processing, 6(2), 136-141. doi:10.1007/s10339-005-0050-6

Montagne, B., Kessels, R. P., De Haan, E. H., \& Perrett, D. I. (2007). The emotion recognition task: A paradigm to measure the perception of facial emotional expressions at different intensities. Perceptual and Motor Skills, 104(2), 589-598. doi:10.2466/pms.104.2.589-598

Moore, D. W., Ashman, T. A., Cantor, J. B., Krinick, R. J., \& Spielman, L. A. (2010). Does gender influence cognitive outcome after traumatic brain injury? Neuropsychological Rehabilitation, 20(3), 340354. doi:10.1080/09602010903250928

Morton, M. V., \& Wehman, P. (1995). Psychosocial and emotional sequelae of individuals with traumatic brain injury: A literature review and recommendations. Brain Injury, 9(1), 81-92.

Mushkudiani, N. A., Engel, D. C., Steyerberg, E. W., Butcher, I., Lu, J., Marmarou, A., . . Maas, A. I. (2007). Prognostic value of demographic characteristics in traumatic brain injury: Results from the IMPACT study. Journal of Neurotrauma, 24(2), 259-269. doi:10. 1089/neu.2006.0028

Neumann, D., McDonald, B. C., West, J., Keiski, M. A., \& Wang, Y. (2015). Neurobiological mechanisms associated with facial affect recognition deficits after traumatic brain injury. Brain Imaging Behavior. doi:10.1007/s11682-0159415-3

Olson, I. R., McCoy, D., Klobusicky, E., \& Ross, L. A. (2013). Social cognition and the anterior temporal lobes: A review and theoretical framework. Social Cognitive and Affective Neuroscience, 8(2), 123 133. doi:10.1093/scan/nss119 
Pettersen, L. (1991). Sensitivity to emotional cues and social behavior in children and adolescents after head injury. Perceptual and Motor Skills, 73(3 Pt, 2), 1139-1150. doi:10.2466/pms.1991.73.3f.1139

Ratcliff, J. J., Greenspan, A. I., Goldstein, F. C., Stringer, A. Y., Bushnik, T., Hammond, F. M., . . W Wright, D. W. (2007). Gender and traumatic brain injury: Do the sexes fare differently? Brain Injury, 21(10), 1023-1030. doi:10.1080/02699050701633072

Rigon, A., Duff, M. C., McAuley, E., Kramer, A., \& Voss, M. W. (2015). ). Is traumatic brain injury associated with reduced inter-hemispheric functional connectivity? A study of large-scale resting state networks following traumatic brain injury. Journal of Neurotrauma. doi:10.1089/ neu. 2014.3847

Rosenberg, H., McDonald, S., Dethier, M., Kessels, R. P., \& Westbrook, R. F. (2014). Facial emotion recognition deficits following moderate-severe traumatic brain tnjury (TBI): Re-examining the valence effect and the role of emotion intensity. Journal of the International Neuropsychological Society, 20(10), 994-1003. doi: $10.1017 / \mathrm{S} 1355617714000940$

Rosenberg, H., Dethier, M., Kessels, R. P., Westbrook, R. F., \& McDonald, S. (2015). Emotion perception after moderatesevere traumatic brain injury: The valence effect and the role of working memory, processing speed, and nonverbal reasoning. Neuropsychology, 29(4), 509-521. doi:10.1037/ neu0000171

Schmidt, A. T., Hanten, G. R., Li, X., Orsten, K. D., \& Levin, H. S. (2010). Emotion recognition following pediatric traumatic brain injury: Longitudinal analysis of emotional prosody and facial emotion recognition. Neuropsychologia, 48(10), 2869-2877. doi:10.1016/j. neuropsychologia.2010.05.029

Shewan, C. M., \& Kertesz, A. (1980). Reliability and validity characteristics of the Western Aphasia Battery (WAB). Journal of Speech and Hearing Disorders, 45(3), 308-324.

Spikman, J. M., Timmerman, M. E., Milders, M. V., Veenstra, W. S., \& van der Naalt, J. (2012). Social cognition impairments in relation to general cognitive deficits, injury severity, and prefrontal lesions in traumatic brain injury patients. Journal of Neurotrauma, 29(1), 101111. doi:10.1089/neu.2011.2084

Temkin, N. R., Corrigan, J. D., Dikmen, S. S., \& Machamer, J. (2009). Social functioning after traumatic brain injury. Journal of Head Trauma Rehabilitation, 24(6), 460-467. doi:10.1097/HTR. 0b013e3181c13413
Turkstra, L. S. (2008). Conversation-based assessment of social cognition in adults with traumatic brain injury. Brain Injury, 22(5), 397-409. doi:10.1080/02699050802027059

Veroude, K., Jolles, J., Croiset, G., \& Krabbendam, L. (2014). Sex differences in the neural bases of social appraisals. Social Cognitive and Affective Neuroscience, 9(4), 513-519. doi:10.1093/scan/nst015

Wehrle, T., Kaiser, S., Schmidt, S., \& Scherer, K. R. (2000). Studying the dynamics of emotional expression using synthesized facial muscle movements. Journal of Personality and Social Psychology, 78(1), $105-119$.

Weisenbach, S. L., Rapport, L. J., Briceno, E. M., Haase, B. D., Vederman, A. C., Bieliauskas, L. A., . . . Langenecker, S. A. (2014). Reduced emotion processing efficiency in healthy males relative to females. Social Cognitive and Affective Neuroscience, 9(3), 316-325. doi:10.1093/scan/nss 137

Welborn, B. L., Papademetris, X., Reis, D. L., Rajeevan, N., Bloise, S. M., \& Gray, J. R. (2009). Variation in orbitofrontal cortex volume: Relation to sex, emotion regulation and affect. Social Cognitive and Affective Neuroscience, 4(4), 328-339. doi:10.1093/scan/nsp028

Williams, C., \& Wood, R. L. (2010). Impairment in the recognition of emotion across different media following traumatic brain injury. Journal of Clinical and Experimental Neuropsychology, 32(2), 113-122. doi:10. 1080/13803390902806543

Willis, M. L., Murphy, J. M., Ridley, N. J., \& Vercammen, A. (2015). Anodal tDCS targeting the right orbitofrontal cortex enhances facial expression recognition. Social Cognitive and Affective Neuroscience, 10(12), 1677-1683. doi:10.1093/scan/nsv057

Wood, W., \& Eagly, A. H. (2000). Once again, the origins of sex differences. American Psychologist, 55(9), 1062-1063.

Wood, R. L., \& Rutterford, N. A. (2006). Demographic and cognitive predictors of long-term psychosocial outcome following traumatic brain injury. Journal of the International Neuropsycholical Society, 12(3), 350-358.

Yim, J., Babbage, D. R., Zupan, B., Neumann, D., \& Willer, B. (2013). The relationship between facial affect recognition and cognitive functioning after traumatic brain injury. Brain Injury, 27(10), 1155-1161. doi:10.3109/02699052.2013.804203

Ylvisaker, M., Turkstra, L. S., \& Coelho, C. (2005). Behavioral and social interventions for individuals with traumatic brain injury: A summary of the research with clinical implications. Seminars in Speech and Language, 26(4), 256-267. doi:10.1055/s-2005-922104 